\title{
Generalized Shortcuts to Adiabaticity and Enhanced Robustness Against Decoherence
}

\author{
Alan C. Santos \& Marcelo S. Sarandy \\ Instituto de Física, Universidade Federal Fluminense, Av. Gal. Milton Tavares de \\ Souza s/n, Gragoatá, 24210-346 Niterói, Rio de Janeiro, Brazil \\ E-mail: msarandy@id.uff.br
}

August 2017

\begin{abstract}
Shortcuts to adiabaticity provide a general approach to mimic adiabatic quantum processes via arbitrarily fast evolutions in Hilbert space. For these counterdiabatic evolutions, higher speed comes at higher energy cost. Here, the counterdiabatic theory is employed as a minimal energy demanding scheme for speeding up adiabatic tasks. As a by-product, we show that this approach can be used to obtain infinite classes of transitionless models, including time-independent Hamiltonians under certain conditions over the eigenstates of the original Hamiltonian. We apply these results to investigate shortcuts to adiabaticity in decohering environments by introducing the requirement of a fixed energy resource. In this scenario, we show that generalized transitionless evolutions can be more robust against decoherence than their adiabatic counterparts. We illustrate this enhanced robustness both for the LandauZener model and for quantum gate Hamiltonians.
\end{abstract}

\section{Introduction}

The adiabatic theorem [1, 2, 3, 4] constitutes a successful strategy for eigenstate tracking in quantum information and quantum control (see, e.g., Ref. [5]). It states that a system initially prepared in an eigenstate of a time-dependent Hamiltonian $H(t)$ will evolve to the corresponding instantaneous eigenstate at a later time $T$, provided that $H(t)$ varies smoothly and that $T$ is much larger than a power of the relevant minimal inverse energy gap. It is worth highlighting that the validity conditions of the adiabatic approximation have been revisited (see, e.g. Refs [6, 7, 8, 9, 10, 11]), which has implied in enhanced formulations of the adiabatic theorem [12, 13] (see also Ref. [14 for a recent review). In a real open-system scenario, the performance of the adiabatic dynamics is upper bounded by the competition between the adiabatic time scale, which is favored by a slow evolution, and the typically short decoherence time scales. This interplay provides an optimal time scale for adiabatic processes in decohering environments [15, 16].

The adiabatic dynamics can be reproduced by generalized transitionless evolutions obtained via shortcuts to adiabaticity [17, 18, 19]. Such accelerated processes allow us to derive an exact adiabatic evolution at an arbitrary finite time. Shortcuts to adiabaticity 
have been used to speed up adiabatic processes in a number of applications, e.g. tracking of many-body systems across quantum phase transitions [20, 21, 22], quantum gate Hamiltonians [23, 24, 25], heat engines in quantum thermodynamics [26], among others (e.g., Refs. [27, 28, 29, 30, 31]). The robustness of such transitionless evolutions has recently been studied through different experimental architectures, as nitrogen-vacancy setups [32], trapped ions [33], atoms in cavities [34, 35], nuclear magnetic resonance (NMR) [36], and optomechanics [37]. Naturally, the speed of the evolution is constrained by the energy cost of the implementation, with faster evolutions being more energy demanding [23]. By providing identical energy resources at a finite evolution time $\tau$, a fundamental problem is then whether shortcuts to adiabaticity can provide a more efficient performance in terms of fidelity than their adiabatic counterparts by adjusting its pace within the decoherence time scales. We address this question by considering a general counter-diabatic theory [38], which is here optimized for a minimum energy consumption. As a by-product, we apply this general approach to obtain infinite classes of transitionless models, including time-independent Hamiltonians under certain conditions over the eigenstates of the original (adiabatic) Hamiltonian. Concerning robustness against decoherence, we consider Markovian open systems and impose fixed energy resources. This is a key point, since unlimited energy provides arbitrarily fast dynamics already for adiabatic evolution, through an arbitrarily large gap between the ground and first excited states. It is then shown that a supremacy of the counter-diabatic dynamics can always be achieved by adjusting the evolution rate. This is illustrated in the Landau-Zener model and in quantum gate Hamiltonians.

\section{Generalized transitionless dynamics theory and energy cost}

The starting point for the counter-diabatic theory is the evolution operator $U(t)$, which can be defined as (see, e.g., Ref. [38])

$$
U(t)=\sum_{n} e^{i \int_{0}^{t} \theta_{n}(\xi) d \xi}\left|n_{t}\right\rangle\left\langle n_{0}\right|,
$$

where $\theta_{n}(t)$ is a set of arbitrary real phases [39, 40] and $\left\{\left|n_{t}\right\rangle=|n(t)\rangle\right\}$ is the set of eigenstates of the original (adiabatic) Hamiltonian $H_{0}(t)$. Let us assume that the quantum system is initially prepared in an specific eigenstate $\left|k_{0}\right\rangle$ of $H_{0}(t)$, namely, $|\psi(0)\rangle=\left|k_{0}\right\rangle$. Then, the Hamiltonian $H_{\mathrm{SA}}(t)=-i U(t) \dot{U}^{\dagger}(t)$, which denotes the shortcut to the adiabatic Hamiltonian $H_{0}(t)$, evolves the system to its instantaneous eigenlevel $|\psi(t)\rangle=e^{i \int_{0}^{t} \theta_{k}(\xi) d \xi}\left|k_{t}\right\rangle$. Explicitly, we write $H_{\mathrm{SA}}(t)$ as $(\hbar=1)$

$$
H_{\mathrm{SA}}(t)=i \sum_{n}\left(\left|\dot{n}_{t}\right\rangle\left\langle n_{t}\left|+i \theta_{n}(t)\right| n_{t}\right\rangle\left\langle n_{t}\right|\right) .
$$

The functions $\theta_{n}(t)$ have originally been identified with the adiabatic phase $\theta_{n}(t)=-E_{n}(t)+i\left\langle n_{t} \mid \dot{n}_{t}\right\rangle$ [41], which exactly mimics an adiabatic evolution. In this particular case, we can write $H_{\mathrm{SA}}(t)$ as $H_{0}(t)+H_{\mathrm{CD}}(t)$, where $H_{0}(t)=$ $\sum_{n} E_{n}(t)\left|n_{t}\right\rangle\left\langle n_{t}\right|$ is the Hamiltonian that drives the adiabatic dynamics and $H_{\mathrm{CD}}(t)=$ $i \sum_{n}\left(\left|\dot{n}_{t}\right\rangle\left\langle n_{t}\left|+\left\langle\dot{n}_{t} \mid n_{t}\right\rangle\right| n_{t}\right\rangle\left\langle n_{t}\right|\right)$ is the counter-diabatic Hamiltonian. 


\subsection{Quantum phases in optimal transitionless quantum driving}

There is a number of situations for which we need not exactly mimic an adiabatic process, but only assure that the system is kept in an instantaneous eigenstate (independently of its associated quantum phase) [23, 24, 25, 26, 27, 28, 29, 32, 33, 34, 37, 42, 43]. This generalized dynamics in terms of arbitrary phases $\theta_{n}(t)$ will be denoted as a transitionless evolution. Now, we will show that $\theta_{n}(t)$ can be nontrivially optimized in transitionless evolutions both in terms of energy cost and robustness against decoherence effects. In this direction, we adopt as a measure of energy cost the average Hilbert-Schmidt norm of the Hamiltonian throughout the evolution, which is given by [44, 23, 45, 46]

$$
\Sigma(\tau)=\frac{1}{\tau} \int_{0}^{\tau}\|H(t)\| d t=\frac{1}{\tau} \int_{0}^{\tau} \sqrt{\operatorname{Tr}\left[H^{2}(t)\right]} d t,
$$

where $\tau$ denotes the total evolution time. The energy cost $\Sigma(\tau)$ aims at identifying changes in the energy coupling constants and gap structure, which typically account for the effort of speeding up adiabatic processes. It is a well-defined measure for finitedimensional Hamiltonians exhibiting non-degeneracies in their energy spectra. Such Hamiltonians describe the quantum systems within the scope of this work. Therefore, Eq. (3) is applicable, e.g., for generic systems composed of a finite number of quantum bits (qubits) under magnetic or electric fields (see Refs. [46, 44] for similar cost measures). Note that Eq. (3) is non-invariant with respect to a change of the zero energy offset. However, by adopting a fixed reference frame, it can be used to quantify the energy cost involved in attempts of accelerating the adiabatic path, either via an increasing of the energy gap in the adiabatic approach or via a reduction of $\tau$ by adjusting the relevant energy couplings in the counter-diabatic theory. In addition, as $\tau$ can be set by the quantum speed limit [47, Eq. (3) allows us to establish a trade-off between speed and energy cost for an arbitrary dynamics [23, 45]. For instance, in NMR experimental setups, the quantity $\|H(s)\|$ represents how intense a magnetic field $\vec{B}(s)$ is expected to be in order to control the speed of such a dynamics.

Now, let us discuss how, for a fixed time $\tau$, the energy cost in a transitionless evolution can be minimized by a suitable choice of the arbitrary parameters $\theta_{n}(t)$. Remarkably, this optimization can be analytically derived, which is established by Theorem 1 below. Its derivation is provided in Appendix A.

Theorem 1. Consider a closed quantum system under adiabatic evolution governed by a Hamiltonian $H_{0}(t)$. The energy cost to implement its generalized transitionless counterpart, driven by the Hamiltonian $H_{S A}(t)$, can be minimized by setting

$$
\theta_{n}(t)=\theta_{n}^{\min }(t)=-i\left\langle\dot{n}_{t} \mid n_{t}\right\rangle .
$$

In particular, for any evolution such that the quantum parallel-transport condition is verified [41], the energy cost to implement a transitionless evolutions is always optimized by choosing $\theta_{n}^{\min }(t)=0$. This approach is useful for providing both realistic and energetically optimal Hamiltonians in several physical scenarios. For example, by 
considering nuclear spins driven by a magnetic field $\vec{B}(t)$ in a nuclear magnetic resonance setup, the energy cost can be optimized by adjusting $\theta_{n}^{\min }(t)$ such that the magnitude $B(t)$ of the magnetic field is reduced, since $\|H(t)\| \propto B(t)$.

As a by-product, the generalized counter-diabatic theory can be used as a tool to yield time-independent Hamiltonians for transitionless evolutions. In general, the Hamiltonian $H_{\mathrm{SA}}(t)$ has its form constrained both by the choice of the phases $\theta_{n}(t)$ and by eigenstates of the adiabatic Hamiltonian $H_{0}(t)$. Thus, we can delineate under what conditions we can choose the set $\left\{\theta_{n}(t)\right\}$ in order to obtain a time-independent Hamiltonian for a transitionless evolution. To answer this question, we impose $\dot{H}_{\mathrm{SA}}(t)=$ 0 considering arbitrary phases $\theta_{n}(t)$. This leads to Theorem 2 below. Its derivation is provided in Appendix B.

Theorem 2. Let $H_{0}(t)$ be a discrete quantum Hamiltonian, with $\left\{\left|m_{t}\right\rangle\right\}$ denoting its set of instantaneous eigenstates. If $\left\{\left|m_{t}\right\rangle\right\}$ satisfies $\left\langle k_{t} \mid \dot{m}_{t}\right\rangle=c_{k m}$, with $c_{k m}$ complex constants $\forall k, m$, then a family of time-independent Hamiltonians $H^{\{\theta\}}$ for generalized transitionless evolutions can be defined by setting $\theta_{m}(t)=\theta$, with $\theta$ a single arbitrary real constant $\forall m$.

\subsection{Transitionless dynamics under decoherence}

Theorems 1 and 2 ensure both an energetically optimal counter-diabatic evolution and families of possible time-independent transitionless Hamiltonians. A rather important point for the generalized counter-diabatic theory is whether it is robust against decoherence. The robustness of the counter-diabatic dynamics and inverse engineering schemes has recently been considered in the literature [48, 49, 50, 51]. Here, in order to provide a comparison between adiabatic and generalized counter-diabatic dynamics, we will require identical energy resources for each implementation. More specifically, we will consider the performance of transitionless evolutions in open systems described by convolutionless master equations given by

$$
d_{s} \rho(s)=-i \tau\left[H_{\mathrm{SA}}(s), \rho(s)\right]+\tau \mathcal{L}_{i}[\rho(s)]
$$

where $\mathcal{L}_{i}[\rho(s)]$ describes the decohering contribution to the quantum dynamics, which is parametrized by the normalized time $s=t / \tau$, with $\tau$ the total time of evolution and $0 \leq s \leq 1$. For Markovian evolution [52, 53], we have $\mathcal{L}_{i}[\rho(s)]=$

$\frac{1}{2} \sum_{i} \gamma_{i}^{2}(s)\left[2 L_{i}(s) \rho(s) L_{i}^{\dagger}(s)-\left\{L_{i}^{\dagger}(s) L_{i}(s), \rho(s)\right\}\right]$, with $L_{i}(s)$ denoting Lindblad operators and $\gamma_{i}(s)$ (positive) decoherence rates. Here, we will consider, as an illustration, Lindblad operators for generalized amplitude damping (GAD) in the eigenbasis of the Hamiltonian, which reads

$$
L_{ \pm}^{\mathrm{GAD}}(s)=U^{\dagger}(s) \sigma_{ \pm} U(s)
$$

where $U(s)$ is the unitary operator that diagonalizes the Hamiltonian and $\sigma_{ \pm}=$ $\left(\sigma_{x} \mp i \sigma_{y}\right) / 2$, with $\left\{\sigma_{x}, \sigma_{y}, \sigma_{z}\right\}$ denoting Pauli matrices. The GAD channel describes dissipation to an environment at finite temperature. Its decoherence rates $\gamma_{+}$and $\gamma_{-}$ 
are given by [54, 55] $\gamma_{+}=\sqrt{\gamma_{0} N_{\mathrm{th}}}$ and $\gamma_{-}=\sqrt{\gamma_{0}\left(N_{\mathrm{th}}+1\right)}$, where $\gamma_{0}$ is the spontaneous emission rate and $N_{\text {th }}$ is the Planck distribution that gives the number of thermal photons at a fixed frequency. For simplicity, we adjust the temperature such that $N_{\text {th }}=1 / 2$ and define $\gamma_{0} \equiv \alpha \omega_{r}$, with $\alpha$ a dimensionless parameter and $\omega_{r}$ some relevant frequency associated with the quantum system. Then, we obtain $\gamma_{+}=\sqrt{\alpha \omega_{r} / 2}$ and $\gamma_{-}=\sqrt{3} \gamma_{+}$. In addition to GAD, we will also consider dephasing in the instantaneous Hamiltonian eigenbasis, whose Lindblad operator reads

$$
L_{\mathrm{d}}(s)=U^{\dagger}(s) \sigma_{z} U(s)
$$

with decoherence rate given by $\gamma_{d} \equiv \alpha \omega_{r}$. Both GAD and dephasing are common decohering processes in a number of physical realizations [53]. They will be used here as probes to the counter-diabatic robustness in the open-system realm.

In this paper we consider that any systematic error due to experimental deviations of fields used to implement the Hamiltonian is negligible. In general, finding an optimal transitionless scheme against arbitrary systematic errors is not a trivial task [56]. In particular, given a fixed class of error, we can obtain an optimal transitionless dynamics for such a class, but the associated dynamics may be not robust against other classes of systematic errors [56, 57, 58].

\section{Transitionless dynamics in the Landau-Zener model}

As a first application, let us consider the dynamics of a two-level quantum system, i.e., a qubit, evolving under the Landau-Zener Hamiltonian

$$
H_{0}^{\mathrm{LZ}}(s)=-\omega\left[\sigma_{z}+\tan \vartheta(s) \sigma_{x}\right]
$$

with $\tan \vartheta(s)$ a dimensionless time-dependent parameter associated with Rabi frequency. This Hamiltonian describes transitions in two-level systems exhibiting anticrossings in its energy spectrum [59]. In particular, it can be applied, e.g., to perform adiabatic population transfer in a two-level system driven by a chirped field [17] and to investigate molecular collision processes [60]. The instantaneous ground $\left|E_{-}(s)\right\rangle$ and first excited $\left|E_{+}(s)\right\rangle$ states of $H_{0}^{\mathrm{LZ}}(s)$ are

$$
\begin{aligned}
& \left|E_{-}(s)\right\rangle=\cos \left[\frac{\vartheta(s)}{2}\right]|0\rangle+\sin \left[\frac{\vartheta(s)}{2}\right]|1\rangle, \\
& \left|E_{+}(s)\right\rangle=-\sin \left[\frac{\vartheta(s)}{2}\right]|0\rangle+\cos \left[\frac{\vartheta(s)}{2}\right]|1\rangle .
\end{aligned}
$$

The system is initialized in the ground state $\left|E_{-}(0)\right\rangle=|0\rangle$ of $H_{0}^{\mathrm{LZ}}(0)$. By considering a unitary dynamics and a sufficiently large total evolution time (adiabatic time), the qubit evolves to the instantaneous ground state $\left|E_{-}(s)\right\rangle$ of $H_{0}^{\mathrm{LZ}}(s)$.

\subsection{Optimal transitionless evolution in Landau-Zener model}

In this section we will discuss the generalized transitionless dynamics theory for

the Landau-Zener model. For optimal energy cost, Eq. (4) establishes $\theta_{n}(t)=$ 
$\left\langle d_{s} E_{ \pm}(s) \mid E_{ \pm}(s)\right\rangle=0$ for the states in Eqs. (9) and (10). Therefore, the optimal Hamiltonian $H_{\mathrm{SA}}(s)$ is given by $H_{\mathrm{SA}}(s)=H_{\mathrm{CD}}^{\mathrm{LZ}}(s)$, with

$$
H_{\mathrm{CD}}^{\mathrm{LZ}}(s)=i \sum_{k= \pm}\left|d_{s} E_{k}(s)\right\rangle\left\langle E_{k}(s)\right|=\frac{d_{s} \vartheta(s)}{2 \tau} \sigma_{y} .
$$

From Eq. (11), we can see that $H_{0}^{\mathrm{LZ}}(s)$ satisfies the hypotheses of Theorem 2 if, and only if, we choose the linear interpolation $\vartheta(s)=\vartheta_{0} s$. Thus, we adopt this choice for simplicity and, consequently, we have $H_{\mathrm{SA}}(s)=\left(\vartheta_{0} / 2 \tau\right) \sigma_{y}$. We observe that a complete (avoided) level crossing picture for the Landau-Zener model is described by varying the parameter $\tan \vartheta(s)$ from $-\infty$ to $+\infty$. Here, we are taking a narrower range for $\tan \vartheta(s)$, which simplifies the description of the transitionless dynamics for the model.

\subsection{Energy cost for the Landau-Zener model}

Now we will be interested in the performance of the transitionless evolution with optimal energy resource so that we impose $\theta_{n}(s)$ as in the Theorem 1. Thus, by considering adiabatic evolution through the Hamiltonian $H_{0}^{\mathrm{LZ}}(s)$, Theorem 1 establishes that the optimal energy resource is performed by setting $\theta_{n}(s)=0$. Considering the energy cost as provided by Eq. (3), we get

$$
\begin{aligned}
& \Sigma_{\mathrm{Ad}}(\tau)=\sqrt{2}|\omega| \int_{0}^{1}|\sec [\vartheta(s)]| d s, \\
& \Sigma_{\mathrm{SA}}(\tau)=\int_{0}^{1} \frac{\left|d_{s} \vartheta(s)\right|}{\sqrt{2} \tau} d s=\frac{|\vartheta(1)|}{\sqrt{2} \tau} .
\end{aligned}
$$

where $\Sigma_{\mathrm{Ad}}$ and $\Sigma_{\mathrm{SA}}$ are the energy costs for the adiabatic and optimal shortcut Hamiltonians, respectively. Remarkably, from Eqs. (12) and (13), it follows that the energy cost for the counter-diabatic Landau-Zener model is independent of the path followed by the system on the Bloch sphere, while its adiabatic counterpart depends on it. In particular, for obtaining $\Sigma_{\mathrm{SA}}(\tau)$, we have used $\tan \vartheta(0)=0$ and, therefore, $\vartheta(0)=0$. Note that there is a range of values for $\tau$ for which the energy cost of the generalized transitionless dynamics is less than its adiabatic version. Indeed, by evaluating the relation between $\Sigma_{\mathrm{Ad}}(\tau)$ and $\Sigma_{\mathrm{SA}}(\tau)$ we get

$$
\mathcal{R}(\tau)=\frac{\Sigma_{\mathrm{Ad}}(\tau)}{\Sigma_{\mathrm{SA}}(\tau)}=|\omega| \tau \frac{2 \int_{0}^{1}|\sec [\vartheta(s)]| d s}{|\vartheta(1)|} .
$$

By imposing identical energy cost, i.e. $\mathcal{R}(\tau)=1$, we obtain

$$
|\omega| \tau=\frac{|\vartheta(1)|}{2 \int_{0}^{1}|\sec [\vartheta(s)]| d s}
$$

Therefore, identical energy cost can be obtained by adjusting $\omega$ according to the total evolution time $\tau$, as in Eq. (14). 


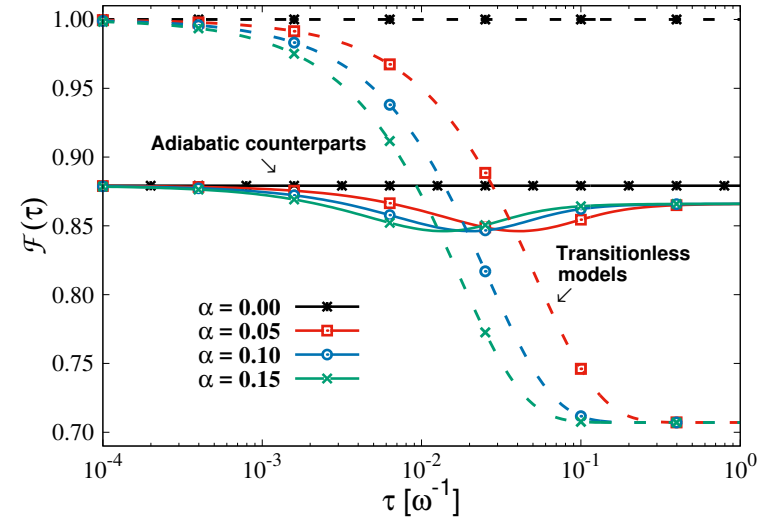

(a)

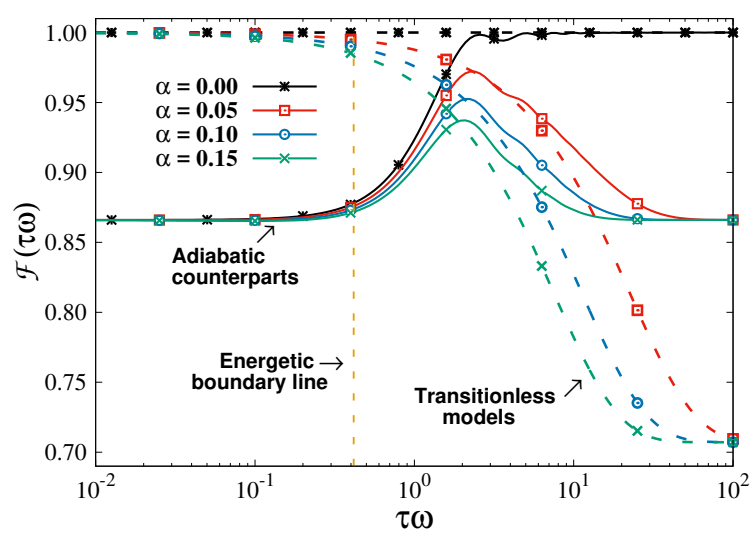

(c)

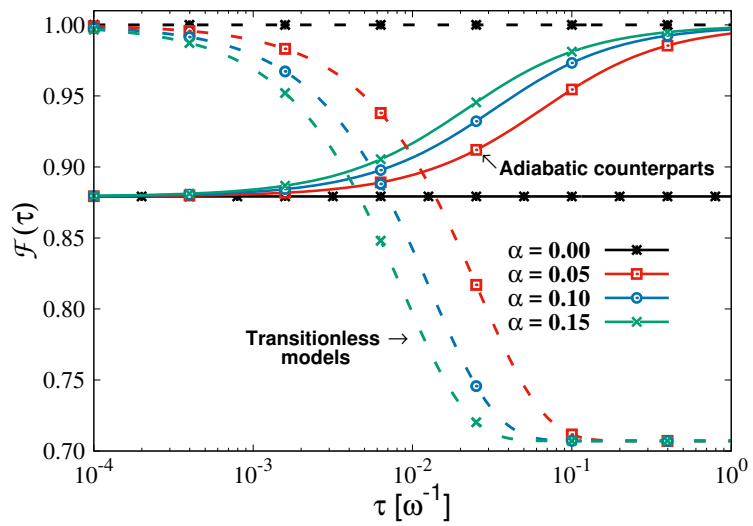

(b)

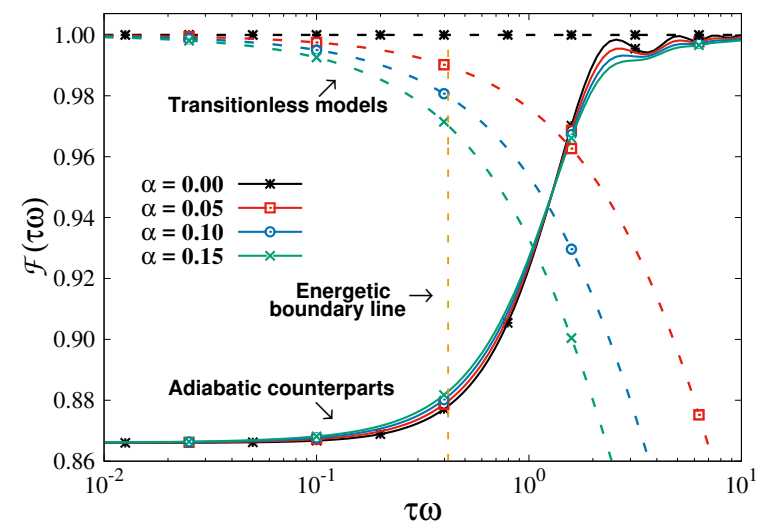

(d)

Figure 1. (Color online) Fidelity $\mathcal{F}(\tau)$ under decoherence in the eigenstate basis for both adiabatic (solid curves) and optimal transitionless dynamics (dashed curves) in the Landau-Zener model. Left column: Fidelity $\mathcal{F}(\tau)$ under GAD for 1a identical and $11 c$ different energy resources. Right column: Fidelity $\mathcal{F}(\tau)$ under dephasing for (1b) identical and (1d) different energy resources. The vertical dashed line in $1 \mathrm{cc}$ and (1d) represents the boundary line between $\Sigma_{\mathrm{Ad}}(\tau) \leqslant \Sigma_{\mathrm{SA}}(\tau)$ and $\Sigma_{\mathrm{Ad}}(\tau) \geqslant \Sigma_{\mathrm{SA}}(\tau)$. We set $\vartheta_{0}=\pi / 3$.

\subsection{Robustness against decoherence in the Landau-Zener model}

We are now ready to compare the behavior under decoherence of both transitionless and adiabatic models. The system is prepared in the ground state $\left|E_{-}(0)\right\rangle=|0\rangle$ of the Landau-Zener Hamiltonian $H_{0}^{\mathrm{LZ}}(s)$ at $s=0$. Then, we let the system evolve aiming at the target state $\left|E_{-}(1)\right\rangle$. We adopt the fidelity $\mathcal{F}(\tau)=\sqrt{\left\langle E_{-}(1)|\rho(1)| E_{-}(1)\right\rangle}$ as a success measure of each protocol, with $\rho(1)$ denoting the solution of Eq. (5) at $s=1$. To settle the problem in a fair scenario, we shall submit both models to the same requirements of energy cost and total evolution time $\tau$. The robustness of adiabatic and optimal transitionless evolutions under GAD and dephasing, for the same and different energetic resources, are shown in Fig. 1. To both situations the decoherence rate strength is controlled by the dimensionless parameter $\alpha$. For equal energy resource provided for 
adiabatic and generalized transitionless evolutions, $\omega_{r}$ will be taken as follows. We consider a set $\left\{\tau_{i} \mid 1 \leq i \leq n\right\}$ of total evolution times. The total time $\tau_{i}$ fixes the energy of the generalized transitionless evolution, with faster evolutions related to shorter times. For a given $\tau_{i}$, we adjust the corresponding frequency $\omega_{i}$ of the Hamiltonian that drives the adiabatic evolution so that $\Sigma_{\mathrm{Ad}}\left(\tau_{i}\right)=\Sigma_{\mathrm{SA}}\left(\tau_{i}\right)$, with $\Sigma_{\mathrm{Ad}}\left(\tau_{i}\right)$ denoting the energy cost of the adiabatic model. The relevant frequency $\omega_{r}$ that sets the decoherence rates $\gamma_{ \pm}$ will then be defined by the average of $\omega_{i}$ for the values of $\tau_{i}$ considered. More specifically, $\omega_{r} \equiv \frac{1}{n} \sum_{i=1}^{n} \omega_{i}$, with $n=200$ in our numerical treatment.

By considering the situation of identical energy cost [see Figs. (1a) and (1b)], the fidelity for unitary dynamics $(\alpha=0)$ in the adiabatic model is constant and smaller than one. This is because of the requirement of fixed energy given by Eq. (15), which imposes a fixed relationship between $\tau$ and $\omega$. The relation between $\tau$ and $\omega$ keeps the adiabatic condition unchanged as we increase $\tau$, since we will have to decrease $\omega$ at the same pace. On the other hand, transitionless evolutions have fidelity close to 1 , since they are not ruled by the adiabatic constraint. For non-unitary evolutions $(\alpha>0)$, generalized transitionless evolutions are more robust than their adiabatic counterparts for any value of $\alpha$ within a range of values for $\tau$. Note also that, for the GAD channel in Fig. (1a), fidelity decreases for intermediate times due to the population of excited states in a thermal environment and then is favored for long times due to the spontaneous emission effect in the energy eigenbasis. In particular, it approximates to the adiabatic fidelity for closed systems for $\tau \rightarrow \infty$. Remarkably, the fidelity of the adiabatic curves increases under dephasing in the eigenstate basis, as shown in Fig. (1b). For this specific case, this occurs due to the fact that the ground eigenprojection $\left|E_{-}(s)\right\rangle\left\langle E_{-}(s)\right|$ is an eigenstate of the Lindblad superoperator, which governs the adiabatic approximation in open quantum systems [15, 16]. Since adiabaticity is governed by the eigenvalue scale of the Lindblad superoperator instead of the Hamiltonian eigenvalue scale, Eq. (15) does not prevent the increase of the adiabatic fidelity as it happens in the closed case. Indeed, decoherence enhances adiabaticity in this situation.

Similar results are also shown in Figs. (1c) and (1 d), where we allow for different resource contents. For this case, the relevant frequency is simply adopted as $\omega_{r} \equiv \omega$. Observe that the behavior of the fidelity curve on the right and left hand side of the vertical line shows that, even for more energy provided for the adiabatic model, the transitionless dynamics can be more robust than the adiabatic dynamics for a fixed $\alpha$. Therefore, generalized transitionless evolutions can be more robust in a real open-system scenario even in situations for which the adiabatic implementation has more energy resource available. For all situations considered in Fig. 1, the crossing points delimit the supremacy region of the optimal transitionless dynamics. This region depends of the coupling strength between the qubit and its reservoir (as measured by the parameter $\alpha)$. Therefore, in general, the advantage of the optimal transitionless evolution is a non-trivial problem, which depends on both the decoherence channel and the coupling strength with the reservoir.

We observe that the generalized counter-diabatic theory can be shown to be more 
robust than its adiabatic counterpart for different values of temperature, with the the choice $N_{\text {th }}=1 / 2$ just accounting for a simple numerical instance. In particular, the plots for each value of the parameter $\alpha$ in Fig. 1 already indicate that the advantage holds for distinct temperature regimes. More specifically, provided the expression for the parameters $\gamma_{+}$and $\gamma_{-}$in terms both of the decoherence rate $\gamma_{0}$ and the temperature (which is implicit in $N_{\text {th }}$ ), we can think of the different values for the parameter $\alpha$ either as a change in the decoherence rate $\gamma_{0}$ (keeping $N_{\text {th }}$ fixed) or as a change in the temperature parameter $N_{\text {th }}$ (keeping $\gamma_{0}$ fixed). Therefore, different values of $\alpha$ can be taken as yielded by a change in the temperature of the bath.

\section{Transitionless dynamics in the counter-diabatic gate model}

Shortcuts to adiabaticity can be used to speed up adiabatic quantum gates. More specifically, they have been applied to perform universal quantum computation (QC) via either counter-diabatic controlled evolutions [23] or counter-diabatic quantum teleportation [24]. As hybrid models, these approaches provide a convenient digital architecture for physical realizations while potentially keeping both the generality and some inherently robustness of analog implementations. Experimentally, digitized implementations of quantum annealing processes have been recently provided [61], with controlled quantum gates adiabatically realized with high fidelity via superconducting qubits [62]. In this Section, by focusing on controlled evolutions, we will now show that counter-diabatic QC can be more robust against decoherence than its adiabatic counterpart as long as the gate runtime is suitably determined within a range of evolution times.

\subsection{Adiabatic and counter-diabatic controlled quantum dynamics}

Consider a bipartite system composed by a target subsystem $\mathcal{T}$ and an auxiliary subsystem $\mathcal{A}$, whose individual Hilbert spaces $\mathcal{H}_{\mathcal{T}}$ and $\mathcal{H}_{\mathcal{A}}$ have dimensions $d_{\mathcal{T}}$ and $d_{\mathcal{A}}$, respectively. The auxiliary subsystem $\mathcal{A}$ will be driven by a family of time-dependent Hamiltonians $\left\{H_{k}(s)\right\}$, with $0 \leq k \leq d_{\mathcal{T}}-1$. The target subsystem will be evolved by a complete set $\left\{P_{k}\right\}$ of orthogonal projectors over $\mathcal{T}$, which satisfy $P_{k} P_{m}=\delta_{k m} P_{k}$ and $\sum_{k} P_{k}=\mathbb{1}$. In a controlled adiabatic dynamics, the composite system $\mathcal{T} \mathcal{A}$ will be governed by a Hamiltonian in the form 63]

$$
H(s)=\sum_{k} P_{k} \otimes H_{k}(s)
$$

with $H_{k}(s)=g(s) H_{k}^{(f)}+f(s) H^{(b)}$, where $H^{(b)}$ is the beginning Hamiltonian, $H_{k}^{(f)}$ is the contribution $k$ to the final Hamiltonian, and the time-dependent functions $f(s)$ and $g(s)$ satisfy the boundary conditions $f(0)=g(1)=1$ and $g(0)=f(1)=0$.

Suppose now we prepare $\mathcal{T} \mathcal{A}$ in the initial state $\left|\Psi_{\text {init }}\right\rangle=|\psi\rangle \otimes\left|\varepsilon_{b}\right\rangle$, where $|\psi\rangle$ is an arbitrary state of $\mathcal{T}$ and $\left|\varepsilon_{b}\right\rangle$ is the (non-degenerate) ground state of $H^{(b)}$. Then $\left|\Psi_{\text {init }}\right\rangle$ is the ground state of the initial Hamiltonian $\mathbb{1} \otimes H^{(b)}$. By applying the adiabatic 
theorem [3, 7], a sufficiently slowing-varying evolution of $H(t)$ will drive the system (up to a phase) to the final state

$$
\left|\Psi_{\text {final }}\right\rangle=\sum_{k} P_{k}|\psi\rangle \otimes\left|\varepsilon_{k}\right\rangle
$$

where $\left|\varepsilon_{k}\right\rangle$ is the ground state of $H_{k}^{(f)}$ [63]. Note that an arbitrary projection $P_{k}$ over the unknown state $|\psi\rangle$ can be yielded by performing a convenient measurement over $\mathcal{A}$. In particular, as will be shown in Subsection 4.2, by suitably designing the auxiliary Hamiltonians $H_{k}(s)$, such a dynamics can be used to adiabatically implement individual quantum gates.

The counter-diabatic version of this controlled evolution has been built in Ref. [23], where it is shown that the transitionless Hamiltonian for the composite system $\mathcal{T} \mathcal{A}$ reads

$$
H_{\mathrm{SA}}(s)=\sum_{k} P_{k} \otimes H_{\mathrm{SA}, k}(s)
$$

where $H_{\mathrm{SA}, k}(s)$ is the piecewise Hamiltonian implementing the shortcut to adiabaticity for the controlled dynamics.

\subsection{Quantum computation via adiabatic controlled evolutions}

Universal sets of quantum gates can be implemented through a bipartite system $\mathcal{T} \mathcal{A}$ composed by a target subsystem $\mathcal{T}$ and a single-qubit auxiliary system $\mathcal{A}$. In our protocol, the target system works as our quantum processor, with any computation performed on it. In others words, both the input and output state, as well as any intermediate stage of the computation, should be encoded in the target system. For example, the target system for a two-qubit gate is composed by the target qubit and the control qubit. On the other hand, the auxiliary qubit works as an ancilla qubit. Any result of measurements over such a qubit is not relevant for the computation result, but it is important for determining whether or not the computation has been successfully realized. Differently from the target system, any information encoded in the auxiliary system may be deleted after the measure. Therefore, quantum gates will be applied to the target subsystem, as a result of a measurement performed on the auxiliary qubit.

Let us begin by considering $\mathcal{T}$ as a single qubit and a single-qubit gate as an arbitrary rotation of angle $\phi$ around a direction $\hat{n}$ over the Bloch sphere. Under this consideration, the Hamiltonian that adiabatically implements such a single-qubit gate for an arbitrary input state $|\psi\rangle=a|0\rangle+b|1\rangle$, with $a, b \in \mathbb{C}$, is given by [63]

$$
H_{\mathrm{sg}}(s)=P_{+} \otimes H_{0}(s)+P_{-} \otimes H_{\phi}(s),
$$

where $\left\{P_{ \pm}\right\}$is a complete set of orthogonal projectors over the Hilbert space of the target qubit. The projectors can be parametrized as $P_{ \pm}=(\mathbb{1} \pm \hat{n} \cdot \vec{\sigma}) / 2$, with $\hat{n}$ associated with the direction of the target qubit on the Bloch sphere. In Eq. (19), each Hamiltonian $H_{\xi}(s)(\xi=\{0, \phi\})$ acts on $\mathcal{A}$, and is given by [63]

$$
H_{\xi}(s)=-\omega\left\{\sigma_{z} \cos \left(\varphi_{0} s\right)+\sin \left(\varphi_{0} s\right)\left[\sigma_{x} \cos \xi+\sigma_{y} \sin \xi\right]\right\}
$$




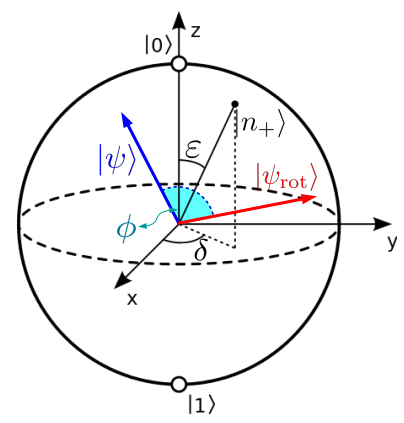

Figure 2. (Color online) Geometric representation of an arbitrary single qubit gate implemented through an adiabatic controlled evolution. Information about the quantum gate to be implemented is encoded in the angles $\varepsilon$ and $\delta$ that set the vector $\left|n_{+}\right\rangle$and in the angle $\phi$ that sets the Hamiltonian in Eq. 20).

with $\varphi_{0}$ denoting an arbitrary parameter that sets the success probability of obtaining the desired state at the end of the evolution. This parameter plays a role in the energy performance of counter-diabatic QC, with probabilistic counter-diabatic QC $\left(\varphi_{0} \neq \pi\right)$ being energetically more favorable than its deterministic $\left(\varphi_{0}=\pi\right)$ counterpart [25]. The projectors $\left\{P_{ \pm}\right\}$may be written in terms of two basis vectors $\left\{\left|n_{ \pm}\right\rangle\right\}$in the Bloch sphere as $\left\{P_{ \pm}\right\}=\left|n_{ \pm}\right\rangle\left\langle n_{ \pm}\right|$, where

$$
\begin{aligned}
& \left|n_{+}\right\rangle=\cos (\varepsilon / 2)|0\rangle+e^{i \delta} \sin (\varepsilon / 2)|1\rangle \\
& \left|n_{-}\right\rangle=-\sin (\varepsilon / 2)|0\rangle+e^{i \delta} \cos (\varepsilon / 2)|1\rangle .
\end{aligned}
$$

Thus, a quantum gate is encoded as a rotation of $\phi$ around the vector $\left|n_{+}\right\rangle$, as shown in the Fig. 2. Now, by expressing the state $|\psi\rangle$ in the basis $\left\{\left|n_{ \pm}\right\rangle\right\}$, we write $|\psi\rangle=\alpha\left|n_{+}\right\rangle+\beta\left|n_{-}\right\rangle$, with $\left|\hat{n}_{ \pm}\right\rangle$being a state in the direction $\hat{n}$ and $\alpha, \beta \in \mathbb{C}$. We then prepare the system in the initial state $|\Psi(0)\rangle=|\psi\rangle|0\rangle$. Then, assuming an adiabatic dynamics, the evolved state $|\Psi(s)\rangle$ is given by the superposition

$$
\begin{aligned}
|\Psi(s)\rangle & =\alpha\left|n_{+}\right\rangle\left|E_{-, 0}(s)\right\rangle+\beta\left|n_{-}\right\rangle\left|E_{-, \phi}(s)\right\rangle \\
& =\cos \left(\frac{\varphi_{0} s}{2}\right)|\psi\rangle|0\rangle+\sin \left(\frac{\varphi_{0} s}{2}\right)\left|\psi_{\text {rot }}\right\rangle|1\rangle,
\end{aligned}
$$

with $\left|\psi_{\text {rot }}\right\rangle=\alpha\left|n_{+}\right\rangle+e^{i \phi} \beta\left|n_{-}\right\rangle$being the rotated desired state and the ground $\left|E_{-, \xi}(s)\right\rangle$ and first excited $\left|E_{+, \xi}(s)\right\rangle$ states of $H_{\xi}(s)$ given by

$$
\begin{aligned}
& \left|E_{-, \xi}(s)\right\rangle=\cos \left(\varphi_{0} s / 2\right)|0\rangle+e^{i \xi} \sin \left(\varphi_{0} s / 2\right)|1\rangle, \\
& \left|E_{+, \xi}(s)\right\rangle=-\sin \left(\varphi_{0} s / 2\right)|0\rangle+e^{i \xi} \cos \left(\varphi_{0} s / 2\right)|1\rangle .
\end{aligned}
$$

We observe that, due to the dynamics of the auxiliary qubit through two adiabatic paths, there are quantum phases $\vartheta_{0}(s)$ and $\vartheta_{\phi}(s)$ accompanying the evolutions associated with $\left|E_{-, 0}(s)\right\rangle$ and $\left|E_{-, \phi}(s)\right\rangle$, respectively. Then, relative phases should in principle be considered in Eq. 223. However, as shown in the Ref. [63], such phases satisfy $\vartheta_{0}(s)=\vartheta_{\phi}(s)$. Thus, they factorize as a global phase of the state $|\Psi(s)\rangle$. At the end of the evolution, a measurement on the auxiliary qubit yields the rotated state with probability $\sin ^{2}\left(\varphi_{0} / 2\right)$ and the input state with probability $\cos ^{2}\left(\varphi_{0} / 2\right)$. The 

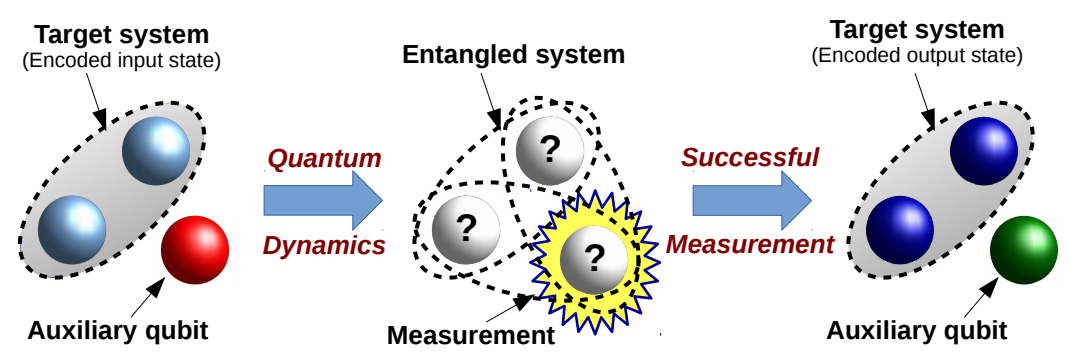

Figure 3. (Color online) Protocol for a probabilistic implementation of a controlled evolution in a two-qubit state. Before the quantum evolution (either adiabatic or nonadiabatic) the input state is encoded in the target system. After the evolution, a measurement (in computational basis) is performed on the auxiliary qubit. A successful measurement corresponds to $|1\rangle$. If the result is $|0\rangle$, the system returns to its initial state and a repetition of the process is required (until $|1\rangle$ is obtained as a result of the measurement).

computation process is therefore probabilistic, which succeeds if the auxiliary qubit ends up in the state $|1\rangle$. Otherwise, the target system automatically returns to the input state and we simply restart the protocol. In the adiabatic scenario, the parameter $\varphi_{0}$ can then be adjusted in order to obtain the optimal fidelity 1 by taking the limit $\varphi_{0} \rightarrow \pi$, implying in a deterministic computation.

This model can be easily adapted to implement controlled single-qubit gates. To this end, the target system has to be increased from one qubit to two qubits, as shown in the scheme provided in Fig. 3. Here, we adopt that the single-qubit gate acts on the target register if the state of the control register is $|1\rangle$. With this convention, the Hamiltonian that implements a controlled single-qubit gate is given by

$$
H_{\mathrm{cg}}(s)=\left(\mathbb{1}-P_{1,-}\right) \otimes H_{0}(s)+P_{1,-} \otimes H_{\phi}(s),
$$

where now the set the orthogonal projectors is given by $P_{k, \pm}=|k\rangle\left\langle k|\otimes| n_{ \pm}\right\rangle\left\langle n_{ \pm}\right|$, where $|k\rangle$ denotes the computational basis. The input state of the target system is now written as $\left|\psi_{2}\right\rangle=a|00\rangle+b|01\rangle+c|10\rangle+d|11\rangle$, with $a, b, c, d \in \mathbb{C}$ and $|n m\rangle=|n\rangle|m\rangle$ denoting the control and target register, respectively. By rewriting $\left|\psi_{2}\right\rangle$ in terms of the basis $\left|n_{ \pm}\right\rangle$, we have $\left|\psi_{2}\right\rangle=\alpha\left|0 n_{+}\right\rangle+\beta\left|0 n_{-}\right\rangle+\gamma\left|1 n_{+}\right\rangle+\delta\left|1 n_{-}\right\rangle$, with $\alpha, \beta, \gamma, \delta \in \mathbb{C}$. Therefore, by assuming adiabatic evolution, the system evolves from the state $\left|\Psi_{2}(0)\right\rangle=\left|\psi_{2}\right\rangle|0\rangle$, to the instantaneous state

$$
\begin{aligned}
|\Psi(s)\rangle= & \alpha\left|0 n_{+}\right\rangle\left|E_{-, 0}(s)\right\rangle+\beta\left|0 n_{-}\right\rangle\left|E_{-, 0}(s)\right\rangle \\
& +\gamma\left|1 n_{+}\right\rangle\left|E_{-, 0}(s)\right\rangle+\delta\left|1 n_{-}\right\rangle\left|E_{-, \phi}(s)\right\rangle \\
= & \cos \left(\frac{\varphi_{0} s}{2}\right)\left|\psi_{2}\right\rangle|0\rangle+\sin \left(\frac{\varphi_{0} s}{2}\right)\left|\psi_{2 \mathrm{rot}}\right\rangle|1\rangle
\end{aligned}
$$

with $\left|\psi_{2 \text { rot }}\right\rangle=\alpha\left|0 n_{+}\right\rangle+\beta\left|0 n_{-}\right\rangle+\gamma\left|1 n_{+}\right\rangle+e^{i \phi} \delta\left|1 n_{-}\right\rangle$being the rotated desired state. We then see that the final state $|\Psi(1)\rangle$ allows for a probabilistic interpretation for the evolution and, consequently, the computation protocol can again be taken as probabilistic $\left(\varphi_{0} \neq \pi\right)$ or deterministic $\left(\varphi_{0}=\pi\right)$. 


\subsection{Quantum computation via counter-diabatic controlled evolutions}

Let us now provide energetically optimal shortcuts to the adiabatic controlled dynamics previously introduced. The transitionless evolution for the quantum gate Hamiltonian $H_{\mathrm{sg}}(s)$ is based on the Hamiltonian $H_{\xi}(s)$ as in Eq. 20), such that for single-qubit and controlled single-qubit gates we have 23

$$
\begin{aligned}
& H_{\mathrm{sg}}^{\mathrm{SA}}=P_{+} \otimes H_{\mathrm{SA}, 0}+P_{-} \otimes H_{\mathrm{SA}, \phi}, \\
& H_{\mathrm{cg}}^{\mathrm{SA}}=\left(\mathbb{1}-P_{1,-}\right) \otimes H_{\mathrm{SA}, 0}+P_{1,-} \otimes H_{\mathrm{SA}, \phi},
\end{aligned}
$$

respectively. Remarkably, each Hamiltonian $H_{\xi}(s)$ satisfies the conditions required by Theorems 1 and 2, so that we can obtain an optimal time-independent Hamiltonian. Thus, the generalized Hamiltonians associated with $H_{\xi}(s)$ for transitionless dynamics can be directly derived from Eq. (2). Notice that, from Eq. (24) and (25), it is possible show that Theorem 2 holds, which implies in

$$
\begin{aligned}
H_{\mathrm{SA}, \xi} & =\frac{1}{\tau} \sum_{k= \pm}\left|d_{s} E_{k, \xi}(s)\right\rangle\left\langle E_{k}(s)\right| \\
& =\frac{\varphi_{0}}{2 \tau}\left[\sigma_{y} \cos \xi-\sigma_{x} \sin \xi\right]
\end{aligned}
$$

where we have used that $\left\langle E_{k, \xi}(s) \mid d_{s} E_{k, \xi}(s)\right\rangle=0$, with $k \in\{+,-\}$. The Hamiltonian in Eq. (30) improves the gate Hamiltonian derived in Ref. [23]. More specifically, Eq. (30) is energetically optimal and given by a time-independent operator.

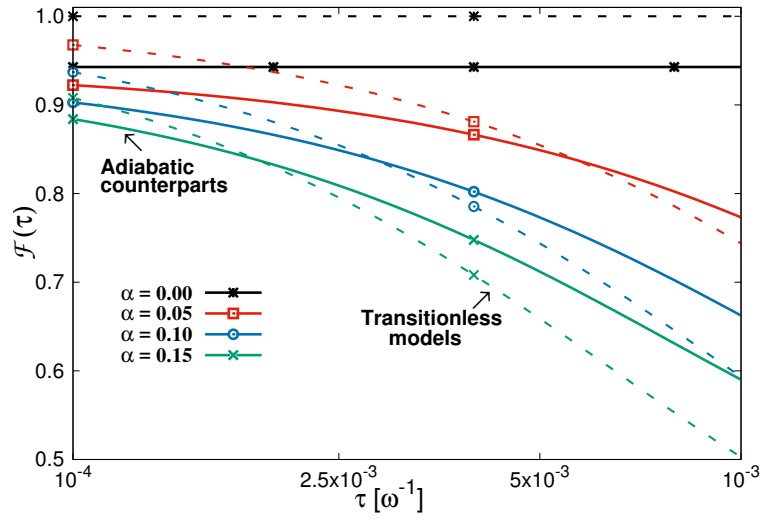

(a)

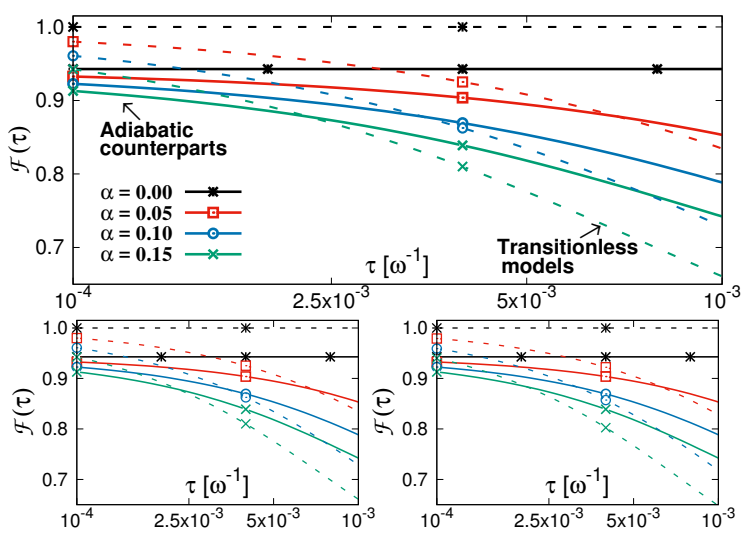

(b)

Figure 4. (Color online) Fidelity $\mathcal{F}(\tau)$ for the implementation of $4 \mathrm{a}$ a CNOT gate to the state $|+\rangle|0\rangle$ and (4b) single qubit gates, provided by (top) a Hadamard gate to the state $|0\rangle$, (bottom left) a phase gate to the state $|+\rangle$, and (bottom right) a $\frac{\pi}{8}$-gate to the state $|+\rangle$. The gates are implemented via deterministic $\left(\varphi_{0}=\pi\right)$ adiabatic QC (solid curves) and probabilistic $\left(\varphi_{0} \approx 0.742 \pi\right)$ counter-diabatic QC (dashed curves), for unitary and non-unitary evolutions under dephasing for identical energy resources. 


\subsection{Energy cost for the quantum gate Hamiltonian}

For a transitionless evolution, it is possible to show that a probabilistic process, with $\varphi_{0} \neq \pi$, is energetically better than the deterministic approach $\varphi_{0}=\pi$ [25]. For this reason, we will consider here the probabilistic model for the generalized counter-diabatic quantum gates. In this scenario, given a fixed amount of energy resource available, our aim is to compare the best adiabatic protocol to implement quantum gates with its best generalized transitionless counterpart. From Eq. (28), we can write the energy cost of a single evolution to implement single-qubit gates

$$
\Sigma_{\mathrm{SA}, \mathrm{sg}}\left(\tau, \varphi_{0}\right)=\frac{\varphi_{0}}{\omega \tau} \Sigma_{\mathrm{sg}}
$$

where $\Sigma_{\mathrm{sg}}=2 \omega$ corresponds to the adiabatic energy cost $\Sigma_{\mathrm{Ad}, \mathrm{sg}}(\tau)$ and $\varphi_{0}$ is the free angle parameter. The energy cost of probabilistic optimal transitionless evolutions can be obtained by defining the quantity

$$
\langle N\rangle \equiv \frac{1}{\sin ^{2}\left(\varphi_{0} / 2\right)},
$$

which is the average number of evolutions for a successful computation. Thus, the average energy cost to implement a probabilistic evolution is [25]

$$
\begin{aligned}
\bar{\Sigma}_{\mathrm{SA}, \mathrm{sg}}\left(\tau, \varphi_{0}\right) & =\langle N\rangle \Sigma_{\mathrm{SA}, \mathrm{sg}}\left(\tau, \varphi_{0}\right) \\
& =\frac{\varphi_{0}}{\omega \tau} \csc ^{2}\left(\varphi_{0} / 2\right) \Sigma_{\mathrm{sg}} .
\end{aligned}
$$

Hence, an optimal scheme requires that the choice of $\varphi_{0}$ is such that $\bar{\Sigma}_{\mathrm{SA}, \mathrm{sg}}\left(\tau, \varphi_{0}\right)$ is minimized. In particular, this minimization is obtained for $\varphi_{0} \approx 0.742 \pi$. It is important mention that the energy cost in Eq. (33) is obtained by two processes: i) energy minimization through the quantum phase $\theta_{n}(t)$ that accompanies the transitionless evolution and ii) application of the probabilistic model of quantum gates.

By considering the energy rate $\mathcal{R}\left(\tau, \varphi_{0}\right)$ for adiabatic and generalized transitionless protocols [similarly as in Eq. (14)], we have

$$
\mathcal{R}\left(\tau, \varphi_{0}\right)=\frac{\Sigma_{\mathrm{Ad}, \mathrm{sg}}(\tau)}{\bar{\Sigma}_{\mathrm{SA}, \mathrm{sg}}\left(\tau, \varphi_{0}\right)}=\frac{\omega \tau}{\varphi_{0}} \sin ^{2}\left(\varphi_{0} / 2\right) .
$$

By imposing identical energy resource, i.e. $\mathcal{R}\left(\tau, \varphi_{0}\right)=1$, we obtain

$$
\omega \tau=\varphi_{0} \csc ^{2}\left(\varphi_{0} / 2\right)
$$

Remarkably, the energy cost for the implementation of a controlled single-qubit gate by the transitionless Hamiltonian in Eq. (29) is simply $\Sigma_{\mathrm{SA}, \mathrm{cg}}=\sqrt{2} \Sigma_{\mathrm{SA}, \mathrm{sg}}$ [23, 25]. The

factor $\sqrt{2}$ also propagates to the adiabatic model, which implies exactly in the same ratio $\mathcal{R}\left(\tau, \varphi_{0}\right)$ and therefore in the same constraint over $\omega \tau$ provided by Eq. (35). 


\subsection{Robustness against decoherence in the quantum gate Hamiltonian}

From Eq. (34), the optimal transitionless quantum gate model will be more efficient from the energy point of view than its adiabatic counterpart for $\omega \tau \geqslant \varphi_{0} \csc ^{2}\left(\varphi_{0} / 2\right)$. Even though this condition solves the problem for closed quantum systems, it is a nontrivial problem the energy efficiency of the generalized transitionless quantum gate Hamiltonian in comparison with its adiabatic version when decoherence effects are not negligible in the physical system. In this section we will study the robustness of counter-diabatic QC by controlled evolutions against decoherence by considering the case of single-qubit gates in addition to the CNOT gate, which constitute a universal set of quantum gates [64. The success of the protocol is measured by the fidelity $\mathcal{F}(\tau)=\sqrt{\left\langle\psi_{\text {rot }}|\rho(1)| \psi_{\text {rot }}\right\rangle}$, with $\rho(1)$ denoting the density operator for the target subsystem, obtained from Eq. (5).

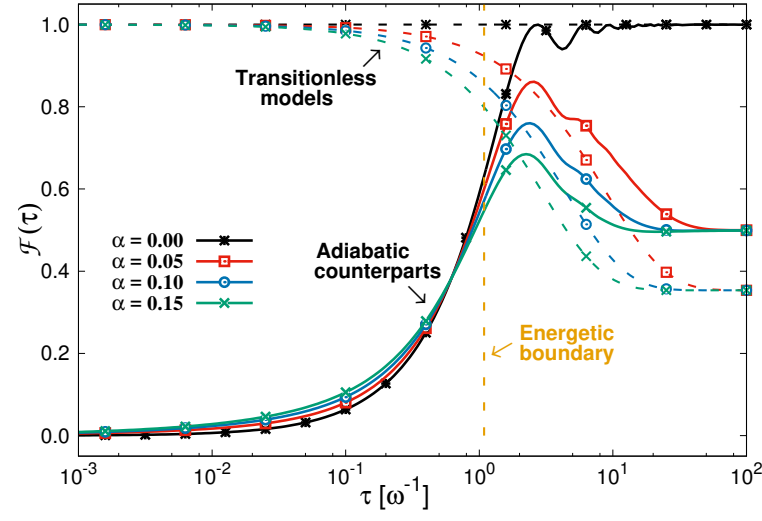

(a)

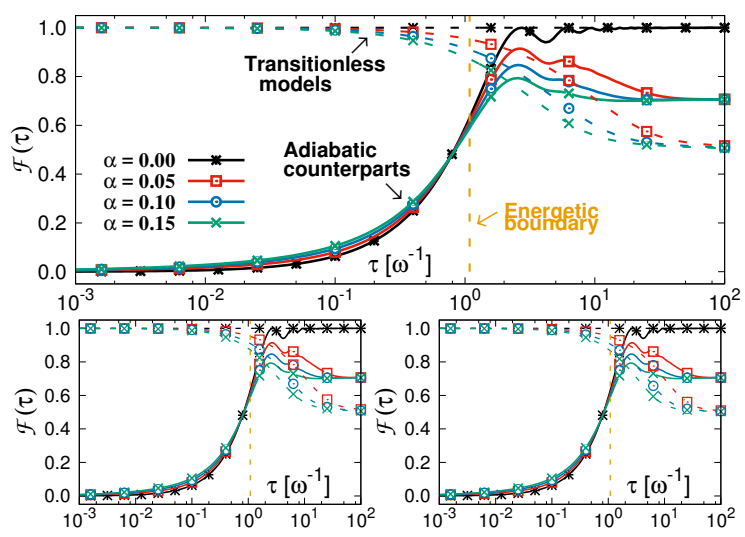

(b)

Figure 5. (Color online) Fidelity $\mathcal{F}(\tau)$ for the implementation of $5 \mathrm{a}$ a CNOT gate to the state $|+\rangle|0\rangle$ and (5b) single qubit gates, provided by (top) a Hadamard gate to the state $|0\rangle$, (bottom left) a phase gate to the state $|+\rangle$, and (bottom right) a $\frac{\pi}{8}$-gate to the state $|+\rangle$. The gates are implemented via deterministic $\left(\varphi_{0}=\pi\right)$ adiabatic QC (solid curves) and probabilistic $\left(\varphi_{0} \approx 0.742 \pi\right)$ counter-diabatic QC (dashed curves), for unitary and non-unitary evolutions under dephasing for different energy resources.

The Hadamard gate is a rotation of $\pi / 2$ around direction $y$ in the Bloch sphere, so that we set $\phi_{\mathrm{Had}}=\pi / 2$ and $\varepsilon_{\mathrm{Had}}=\delta_{\mathrm{Had}}=\pi / 2$ in Eqs. (21) and (22). For the case of phase and $\frac{\pi}{8}$ gates, we take them as rotations around the direction $z$ of an angle $\pi$ and $\pi / 4$, respectively, so that we take $\varepsilon_{\text {pha }}=\varepsilon_{\frac{\pi}{8}}=0, \phi_{\text {pha }}=\pi$, and $\phi_{\frac{\pi}{8}}=\pi / 4$. Concerning the CNOT gate, it is a controlled implementation of the operation $\sigma_{x}$ (flip gate), which can be viewed as a rotation of $\pi$ around the $x$ direction. Thus we set $\varepsilon_{\mathrm{CNOT}}=\pi / 2, \delta_{\mathrm{CNOT}}=0$, and $\phi_{\mathrm{CNOT}}=\pi$. Therefore, from Eq. (30), we have the 


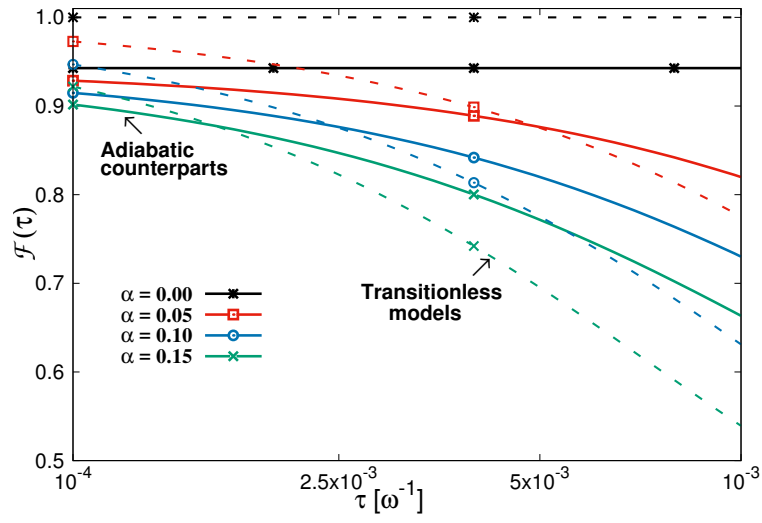

(a)

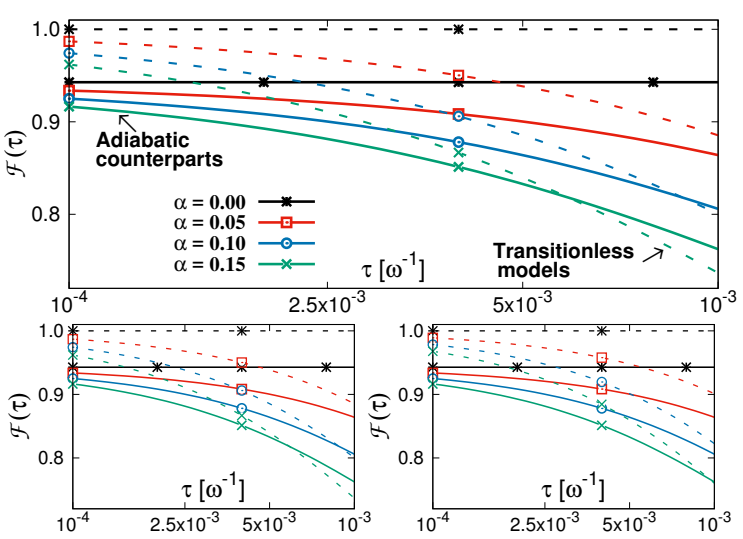

(b)

Figure 6. (Color online) Fidelity $\mathcal{F}(\tau)$ for the implementation of 6a a CNOT gate to the state $|+\rangle|0\rangle$ and (6b) single qubit gates, provided by (top) a Hadamard gate to the state $|0\rangle$, (bottom left) a phase gate to the state $|+\rangle$, and (bottom right) a $\frac{\pi}{8}$-gate to the state $|+\rangle$. The gates are implemented via deterministic $\left(\varphi_{0}=\pi\right)$ adiabatic QC (solid curves) and probabilistic $\left(\varphi_{0} \approx 0.742 \pi\right)$ counter-diabatic QC (dashed curves), for unitary and non-unitary evolutions under GAD for identical energy resources.

following counter-diabatic Hamiltonians

$$
\begin{aligned}
& H_{\mathrm{CD}, 0}=\hbar \frac{\varphi_{0}}{2 \tau} \sigma_{y}, H_{\mathrm{CD}, \frac{\pi}{2}}=-\hbar \frac{\varphi_{0}}{2 \tau} \sigma_{x}, \\
& H_{\mathrm{CD}, 0}=\hbar \frac{\varphi_{0}}{2 \tau} \sigma_{y}, H_{\mathrm{CD}, \pi}=-\hbar \frac{\varphi_{0}}{2 \tau} \sigma_{y}, \\
& H_{\mathrm{CD}, 0}=\hbar \frac{\varphi_{0}}{2 \tau} \sigma_{y}, H_{\mathrm{CD}, \frac{\pi}{4}}=\hbar \frac{\varphi_{0}}{2 \sqrt{2} \tau}\left(\sigma_{y}-\sigma_{x}\right), \\
& H_{\mathrm{CD}, 0}=\hbar \frac{\varphi_{0}}{2 \tau} \sigma_{y}, H_{\mathrm{CD}, \pi}=-\hbar \frac{\varphi_{0}}{2 \tau} \sigma_{y},
\end{aligned}
$$

for Hadamard, phase, $\frac{\pi}{8}$, and CNOT gates, respectively. In order to study the robustness of single qubit gates we have considered the input stats $\left|\psi_{\mathrm{Had}}\right\rangle=|0\rangle$ for Hadamard operation and $\left|\psi_{\text {pha }}\right\rangle=|+\rangle=(1 / \sqrt{2})(|0\rangle+|1\rangle)$ for phase and $\frac{\pi}{8}$-gate. On the other hand, for the CNOT gate, we consider the initial state $|\psi(0)\rangle=|+\rangle|0\rangle$ and apply the gate Hamiltonian to create a Bell state $\left|\psi_{00}\right\rangle=(1 / \sqrt{2})(|00\rangle+|11\rangle)$. Fidelity is then obtained from the explicit solution of the Lindblad equation for $\rho(1)$. For instance, for CNOT, we have $\mathcal{F}(\tau)=\sqrt{\left\langle\psi_{00}|\rho(1)| \psi_{00}\right\rangle}$.

The robustness of the universal set of quantum gate under dephasing is illustrated in Figs. 4 and 5 for identical and different resources imposed, respectively. In these plots, we compare the optimal adiabatic (deterministic) implementation with its optimal transitionless version (probabilistic computation and optimal quantum phases). Note that the generalized transitionless approach shows a higher fidelity for fast dynamics, but there are regimes for which the adiabatic approach shows a better fidelity for a fixed $\alpha$. Energy optimization in the optimal transitionless model is achieved for $\varphi_{0} \approx 0.742 \pi[25]$. Figs. 6 and 7 show similar results result for non-unitary evolution under GAD, with equivalent and different resources provided to the adiabatic and optimal transitionless 


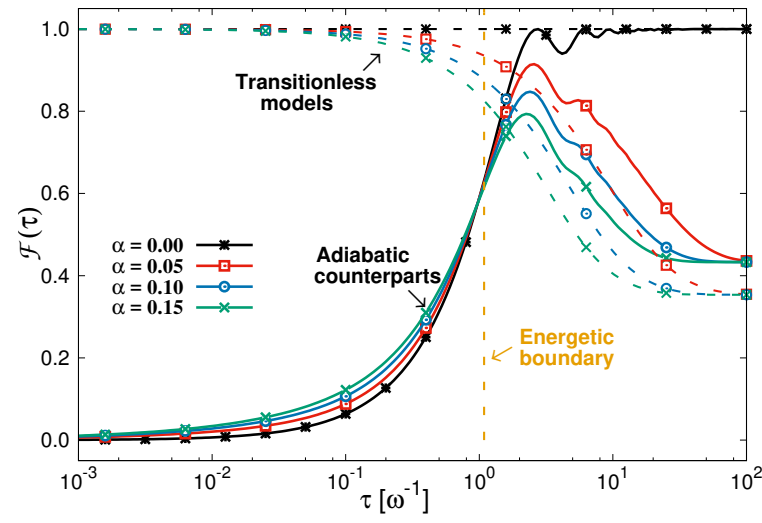

(a)

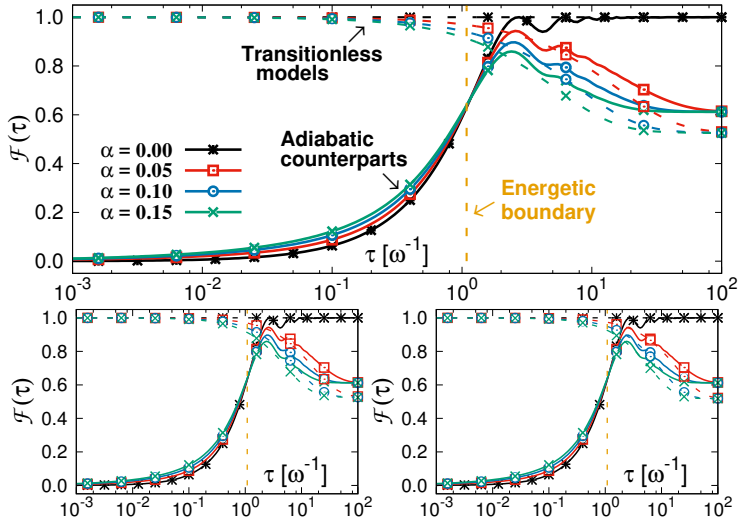

(b)

Figure 7. (Color online) Fidelity $\mathcal{F}(\tau)$ for the implementation of $7 \mathrm{a}$ a CNOT gate to the state $|+\rangle|0\rangle$ and $(7 \mathrm{~b})$ single qubit gates, provided by (top) a Hadamard gate to the state $|0\rangle$, (bottom left) a phase gate to the state $|+\rangle$, and (bottom right) a $\frac{\pi}{8}$-gate to the state $|+\rangle$. The gates are implemented via deterministic $\left(\varphi_{0}=\pi\right)$ adiabatic QC (solid curves) and probabilistic $\left(\varphi_{0} \approx 0.742 \pi\right)$ counter-diabatic QC (dashed curves), for unitary and non-unitary evolutions under GAD for different energy resources.

model, respectively. In any case of decoherence and energetic resource, there always exist dynamical regimes for which the optimal transitionless evolutions are more robust and therefore a preferred approach in a decohering physical environment.

\section{Conclusion}

In summary, we have developed a generalized minimal energy demanding counterdiabatic theory, which is able to yield efficient shortcuts to adiabaticity via fast transitionless evolutions. Moreover, we have investigated the robustness of adiabatic and counter-diabatic dynamics under decoherence by introducing the requirement of fixed energy resources, so that a comparison is settled down in a fair scenario. Then, we have shown both for the Landau-Zener model and for quantum gate Hamiltonians that there always exist dynamical regimes for which generalized transitionless evolutions are more robust and therefore a preferred approach in a decohering setup. This has been shown both for the dephasing and GAD channels acting on the eigenstate bases. It is also possible to show the advantage in other bases, such as the computational basis. The general picture is that the gain will typically occur during some finite time range, disappearing in the limit of long evolution times. These results are encouraging for the generalized transitionless approach in the open-system realm as long as local Hamiltonians are possible to be designed. In the specific case of quantum gate Hamiltonians, this approach can be applied, e.g. to derive robust local building blocks for analog implementations of quantum circuits (see, e.g., Refs. [62, 61]). Experimental realizations, extensions for dealing with systematic errors, and generalized shortcuts via reservoir engineering are further directions left for future research. 


\section{Acknowledgments}

We acknowledge Gonzalo Muga and Tameem Albash for useful discussions. A.C.S. is supported by CNPq-Brazil. M.S.S. acknowledges support from CNPq-Brazil (No. 303070/2016-1), FAPERJ (No. 203036/2016), and the Brazilian National Institute for Science and Technology of Quantum Information (INCT-IQ).

\section{Appendix A. Proof of Theorem 1}

Theorem 1. Consider a closed quantum system under adiabatic evolution governed by a Hamiltonian $H_{0}(t)$. The energy cost to implement its generalized transitionless counterpart, driven by the Hamiltonian $H_{S A}(t)$, can be minimized by setting $\theta_{n}(t)=$ $\theta_{n}^{\min }(t)=-i\left\langle\dot{n}_{t} \mid n_{t}\right\rangle$.

Proof. We adopt as a measure of energy cost the Hamiltonian Hilbert-Schmidt norm, which reads

$$
\Sigma_{\mathrm{SA}}(\tau)=\frac{1}{\tau} \int_{0}^{\tau} \sqrt{\operatorname{Tr}\left[H_{\mathrm{SA}}^{2}(t)\right]} d t
$$

Then, we obtain

$$
\begin{aligned}
& H_{\mathrm{SA}}^{2}(t)=\hbar^{2} \sum_{n}\left[\left|\dot{n}_{t}\right\rangle\left\langle\dot{n}_{t}\left|+\theta_{n}^{2}(t)\right| n_{t}\right\rangle\left\langle n_{t}\right|+\right. \\
& \left.\left.i \theta_{n}(t)\left(\left|n_{t}\right\rangle\left\langle\dot{n}_{t}|-| \dot{n}_{t}\right\rangle\left\langle n_{t}\right|\right]\right)\right] .
\end{aligned}
$$

By taking the trace of $H_{\mathrm{SA}}^{2}(t)$ in Eq. $(\mathrm{A} .2$, we have

$$
\begin{aligned}
& \operatorname{Tr}\left[H_{\mathrm{SA}}^{2}(t)\right]=\sum_{m}\left\langle m_{t}\left|H_{\mathrm{SA}}^{2}(t)\right| m_{t}\right\rangle \\
& =\hbar^{2} \sum_{n}\left[\left\langle\dot{n}_{t} \mid \dot{n}_{t}\right\rangle+\theta_{n}^{2}(t)+2 i \theta_{n}(t)\left\langle\dot{n}_{t} \mid n_{t}\right\rangle\right] .
\end{aligned}
$$

Then

$$
\Sigma_{\mathrm{SA}}(\tau)=\frac{1}{\tau} \int_{0}^{\tau} \sqrt{\sum_{n}\left\langle\dot{n}_{t} \mid \dot{n}_{t}\right\rangle+\Gamma_{n}\left(\theta_{n}\right)} d t,
$$

where we have $\Gamma_{n}\left(\theta_{n}\right)=\theta_{n}^{2}(t)+2 i \theta_{n}(t)\left\langle\dot{n}_{t} \mid n_{t}\right\rangle$.

We can now find out the functions $\theta_{n}(t)$ that minimize the energy cost in transitionless evolutions. For this end, we minimize the quantity $\Sigma_{\mathrm{SA}}(\tau)$ for the Hamiltonian $H_{\mathrm{SA}}(t)$ with respect to parameters $\theta_{n}(t)$, where we will adopt it being independents. By evaluating $\partial_{\theta_{n}} \Sigma(\tau)$, we obtain

$$
\partial_{\theta_{n}} \Sigma_{\mathrm{SA}}(\tau)=\frac{1}{2 \tau} \int_{0}^{\tau} \frac{\partial_{\theta_{n}}\left\{\operatorname{Tr}\left[H_{\mathrm{SA}}^{2}(t)\right]\right\}}{\sqrt{\operatorname{Tr}\left[H^{2}(t)\right]}} d t .
$$

We then impose $\partial_{\theta_{n}}\left\{\operatorname{Tr}\left[H_{\mathrm{SA}}^{2}(t)\right]\right\}=0$ for all time $t \in[0, \tau]$, which ensures $\partial_{\theta_{n}} \Sigma_{\mathrm{SA}}(\tau)=$ 0. Thus, by using Eq. A.3., we write

$$
\partial_{\theta_{n}}\left\{\operatorname{Tr}\left[H_{\mathrm{SA}}^{2}(t)\right]\right\}=2 \theta_{n}(t)+2 i\left\langle\dot{n}_{t} \mid n_{t}\right\rangle=0 .
$$


This implies

$$
\theta_{n}(t)=\theta_{n}^{\min }(t)=-i\left\langle\dot{n}_{t} \mid n_{t}\right\rangle .
$$

From the second derivative analysis, it follows that the choice for $\theta_{n}(t)$ as in Eq. A.7 necessarily minimizes the energy cost, namely, $\left.\partial_{\theta_{n}}^{2} \Sigma_{\mathrm{SA}}(\tau)\right|_{\theta_{n}=\theta_{n}^{\min }}>0$, which concludes the proof.

\section{Appendix B. Proof of Theorem 2}

Theorem 2. Let $H_{0}(t)$ be a discrete quantum Hamiltonian, with $\left\{\left|m_{t}\right\rangle\right\}$ denoting its set of instantaneous eigenstates. If $\left\{\left|m_{t}\right\rangle\right\}$ satisfies $\left\langle k_{t} \mid \dot{m}_{t}\right\rangle=c_{k m}$, with $c_{k m}$ complex constants $\forall k, m$, then a family of time-independent Hamiltonians $H^{\{\theta\}}$ for generalized transitionless evolutions can be defined by setting $\theta_{m}(t)=\theta$, with $\theta$ a single arbitrary real constant $\forall m$.

Proof. By taking the time derivative of the Hamiltonian $H_{\mathrm{SA}}(t)$, we obtain

$$
\dot{H}_{\mathrm{SA}}(t)=i \sum_{n} \frac{d}{d t}\left[\left|\dot{n}_{t}\right\rangle\left\langle n_{t}\left|+i \theta_{n}(t)\right| n_{t}\right\rangle\left\langle n_{t}\right|\right]
$$

Then, the matrix elements of $\dot{H}_{\mathrm{SA}}(t)$ in the eigenbasis $\left\{\left|m_{t}\right\rangle\right\}$ of the Hamiltonian $H_{0}(t)$ read

$$
\begin{aligned}
\left\langle k_{t}\right| \dot{H}_{\mathrm{SA}} & (t)\left|m_{t}\right\rangle=i\left\langle k_{t} \mid \ddot{m}_{t}\right\rangle+i \sum_{n}\left\langle k_{t} \mid \dot{n}_{t}\right\rangle\left\langle\dot{n}_{t} \mid m_{t}\right\rangle \\
& -\left[\dot{\theta}_{k}(t) \delta_{k m}+\theta_{m}(t)\left\langle k_{t} \mid \dot{m}_{t}\right\rangle+\theta_{k}(t)\left\langle\dot{k}_{t} \mid m_{t}\right\rangle\right] .
\end{aligned}
$$

Now, by using $\left\langle k_{t} \mid \dot{n}_{t}\right\rangle=-\left\langle\dot{k}_{t} \mid n_{t}\right\rangle$, we write $\left\langle k_{t} \mid \dot{n}_{t}\right\rangle\left\langle\dot{n}_{t} \mid m_{t}\right\rangle=\left\langle\dot{k}_{t} \mid n_{t}\right\rangle\left\langle n_{t} \mid \dot{m}_{t}\right\rangle$ and thus

$$
\begin{aligned}
& \left\langle k_{t}\left|\dot{H}_{\mathrm{SA}}(t)\right| m_{t}\right\rangle=i \frac{d}{d t}\left[\left\langle k_{t} \mid \dot{m}_{t}\right\rangle\right] \\
& \quad-\left\{\dot{\theta}_{k}(t) \delta_{k m}+\left[\theta_{m}(t)-\theta_{k}(t)\right]\left\langle k_{t} \mid \dot{m}_{t}\right\rangle\right\} .
\end{aligned}
$$

For $k=m$ in Eq. B.2, we impose the vanishing of the diagonal elements of $\dot{H}_{\mathrm{SA}}(t)$, namely, $\left\langle k_{t}\left|\dot{H}_{\mathrm{SA}}(t)\right| k_{t}\right\rangle=0$. This yields

$$
\dot{\theta}_{m}(t)=i \frac{d}{d t}\left[\left\langle m_{t} \mid \dot{m}_{t}\right\rangle\right]
$$

On the other hand, for $k \neq m$ in Eq. (B.2), we now impose the vanishing of the off-diagonal elements of $\dot{H}_{\mathrm{SA}}(t)$, namely, $\left\langle k_{t}\left|\dot{H}_{\mathrm{SA}}(t)\right| m_{t}\right\rangle=0(k \neq m)$. This yields

$$
i \frac{d}{d t}\left[\left\langle k_{t} \mid \dot{m}_{t}\right\rangle\right]=\left[\theta_{m}(t)-\theta_{k}(t)\right]\left\langle k_{t} \mid \dot{m}_{t}\right\rangle \quad(k \neq m)
$$

By taking $\left\langle m_{t} \mid \dot{m}_{t}\right\rangle \equiv c_{m m}$ in Eq. (B.3), with $c_{m m}$ denoting by hypothesis complex constants, we get $\theta_{m}(t)=\theta_{m}(0) \equiv \theta_{m}$, namely, $\theta_{m}(t)$ is a constant function $\forall m$. Moreover, by using $\left\langle k_{t} \mid \dot{m}_{t}\right\rangle \equiv c_{k m}$ in Eq. (B.4), with $c_{k m}$ denoting nonvanishing complex 
constants, we obtain $\theta_{k}=\theta_{m}, \forall k, m$. If $c_{k m}=0$, then $\theta_{k}$ and $\theta_{m}$ are not necessarily equal, but Eq. (B.4) will also be satisfied by this choice. Therefore, it follows that $\theta_{m}(t)$ can be simply taken as

$$
\theta_{m}(t)=\theta_{m}=\theta \quad \forall m
$$

with $\theta$ a single real constant. This concludes the proof.

\section{References}

[1] Born M and Fock V 1928 Zeitschrift für Physik A Hadrons and Nuclei 51165

[2] Kato T 1950 Journal of the Physical Society of Japan 5435

[3] Messiah A 1962 Quantum Mechanics Quantum Mechanics (North-Holland Publishing Company)

[4] Teufel S 2003 Adiabatic perturbation theory in quantum dynamics vol 1821 (Lecture notes in mathematics, Springer-Verlag Berlin Heidelberg)

[5] Jing J, Sarandy M S, Lidar D A, Luo D W and Wu L A 2016 Phys. Rev. A 94(4) 042131

[6] Marzlin K P and Sanders B C 2004 Phys. Rev. Lett. 93160408

[7] Sarandy M S, Wu L A and Lidar D A 2004 Quantum Information Processing 3331

[8] Tong D M, Singh K, Kwek L C, and Oh C H 2005 Phys. Rev. Lett. 95110407.

[9] Du J, Hu L, Wang Y, Wu J, Zhao M and Suter D 2008 Phys. Rev. Lett. 101(6) 060403

[10] Wu J D, Zhao M S, Chen J L and Zhang Y D 2008 Phys. Rev. A 77(6) 062114

[11] Amin M 2009 Phys. Rev. Lett. 102 2204012009).

[12] Ambainis A and Regev O 2004 arXiv:quant-ph/0411152.

[13] Jansen S, Ruskai M B and Seiler R 2007 J. Math. Phys. 48102111

[14] Albash T and Lidar D A 2016 arXiv preprint arXiv:1611.04471

[15] Sarandy M S and Lidar D A 2005 Phys. Rev. A 71(1) 012331

[16] Sarandy M S and Lidar D A 2005 Phys. Rev. Lett. 95(25) 250503

[17] Demirplak M and Rice S A 2003 J. Phys. Chem. A 1079937

[18] Demirplak M and Rice S A 2005 J. Phys. Chem. B 1096838

[19] Berry M 2009 J. Phys. A: Math. Theor. 42365303

[20] del Campo A 2013 Phys. Rev. Lett. 111100502

[21] Saberi H, Opatrný T c v, Mølmer K and del Campo A 2014 Phys. Rev. A 90(6) 060301

[22] Hatomura T 2017 J. Phys. Soc. Jpn. 86094002

[23] Santos A C and Sarandy M S 2015 Sci. Rep. 515775

[24] Santos A C, Silva R D and Sarandy M S 2016 Phys. Rev. A 93(1) 012311

[25] Coulamy I B, Santos A C, Hen I and Sarandy M S 2016 Frontiers in ICT 319 ISSN 2297-198X

[26] Beau M, Jaramillo J and del Campo A 2016 Entropy 18168

[27] Stefanatos D 2014 Phys. Rev. A 90(2) 023811

[28] Lu M, Xia Y, Shen L T, Song J and An N B 2014 Phys. Rev. A 89(1) 012326

[29] Deffner S 2016 New J. Phys. 18012001

[30] Chen Y H, Xia Y, Chen Q Q and Song J 2014 Phys. Rev. A 89(3) 033856

[31] Song X K, Zhang H, Ai Q, Qiu J and Deng F G 2016 New J. Phys. 18023001

[32] Liang Z T, Yue X, Lv Q, Du Y X, Huang W, Yan H and Zhu S L 2016 Phys. Rev. A 93(4) 040305

[33] An S, Lv D, Del Campo A and Kim K 2016 Nat. Commun. 712999

[34] Chen Z, Chen Y, Xia Y, Song J and Huang B 2016 Sci. Rep. 622202

[35] Wu S, Huang X and Yi X 2017 Phys. Rev. A 96042104

[36] Vandermause J and Ramanathan C 2016 Phys. Rev. A 93(5) 052329

[37] Zhang H, Song X K, Ai Q, Zhang M and Deng F G 2016 arXiv:1610.09938

[38] Torrontegui E, Ibáñez S, Martínez-Garaot S, Modugno M, Del Campo A, Guéry-Odelin D, Ruschhaupt A, Chen X and Muga J G 2013 Adv. At. Mol. Opt. Phys 62117

[39] Muga J G, Chen X, Ibáñez S, Lizuain I and Ruschhaupt A 2010 J. Phys. B: At. Mol. Opt. Phys. 43085509 
[40] Chen X, Torrontegui E and Muga J G 2011 Phys. Rev. A 83(6) 062116

[41] Berry M V 1984 Proc. R. Soc. A 39245

[42] Herrera M, Sarandy M S, Duzzioni E I and Serra R M 2014 Phys. Rev. A 89(2) 022323

[43] Chen X, Lizuain I, Ruschhaupt A, Guéry-Odelin D and Muga J G 2010 Phys. Rev. Lett. 105(12) 123003

[44] Zheng Y, Campbell S, De Chiara G and Poletti D 2016 Phys. Rev. A 94(4) 042132

[45] Campbell S and Deffner S 2017 Phys. Rev. Lett. 118(10) 100601

[46] Kieferová M and Wiebe N 2014 New J. Phys. 16123034

[47] Deffner S and Lutz E 2013 J. Phys. A: Math. Theor. 46335302

[48] Kang Y H, Chen Y H, Wu Q C, Huang B H, Xia Y and Song J 2016 Sci. Rep. 630151

[49] Chen Y H, Huang B H, Song J and Xia Y 2016 Opt. Comm. 380 140-147

[50] Liu B J, Huang Z H, Xue Z Y and Zhang X D 2017 Phys. Rev. A 95(6) 062308

[51] Jing J, Wu L A, Sarandy M S and Muga J G 2013 Phys. Rev. A 88(5) 053422

[52] Lindblad G 1976 Comm. Math. Phys. 48119

[53] Breuer H P and Petruccione F 2007 The Theory of Open Quantum Systems (Oxford University Press, Oxford, UK: Cambridge University Press) ISBN 9780199213900

[54] Srikanth R and Banerjee S 2008 Phys. Rev. A 77(1) 012318

[55] Cafaro C and van Loock P 2014 Phys. Rev. A 89(2) 022316

[56] Ruschhaupt A, Chen X, Alonso D and Muga J 2012 New J. Phys. 14093040

[57] Tseng S Y, Wen R D, Chiu Y F and Chen X 2014 Opt. Express 22 18849-18859

[58] Lu X J, Chen X, Ruschhaupt A, Alonso D, Guérin S and Muga J G 2013 Phys. Rev. A 88(3) 033406

[59] Zener C 1932 Proc. R. Soc. A 137696

[60] Lee H and George T F 1979 J. Phys. Chem. 83928

[61] Barends R, Shabani A, Lamata L, Kelly J, Mezzacapo A, Heras U L, Babbush R, Fowler A G, Campbell B, Chen Y, Chen Z, Chiaro B, Dunsworth A, Jeffrey E, Lucero E, Megrant A, Mutus J Y, Neeley M, Neill C, OMalley P J J, Quintana C, Roushan P, Sank D, Vainsencher A, Wenner J, White T C, Solano E, Neven H and Martinis J M 2016 Nature 534222

[62] Martinis J M and Geller M R 2014 Phys. Rev. A 90(2) 022307

[63] Hen I 2015 Phys. Rev. A 91(2) 022309

[64] Barenco A, Bennett C H, Cleve R, DiVincenzo D P, Margolus N, Shor P, Sleator T, Smolin J A and Weinfurter H 1995 Phys. Rev. A 52(5) 3457-3467 